ECOSS-18, Vienna, September 21-24, 1999, submitted to Surface Science

The abstract ID: A9XSGW

\title{
Submonolayer growth with decorated island edges
}

\author{
Miroslav Kotrla f \\ Institute of Physics Academy of Sciences of the Czech Republic, \\ Na Slovance 2, 18221 Praha 8, Czech Republic \\ Joachim Krug \\ Fachbereich Physik, Universität GH Essen, 45117 Essen, Germany \\ Pavel Šmilauer \\ Institute of Physics Academy of Sciences of the Czech Republic, \\ Cukrovarnická 10, 162 53 Praha 6, Czech Republic
}

\begin{abstract}
We study the dynamics of island nucleation in the presence of adsorbates using kinetic Monte Carlo simulations of a two-species growth model. Adatoms (A-atoms) and impurities (B-atoms) are codeposited, diffuse and aggregate subject to attractive AA- and AB-interactions. Activated exchange of adatoms with impurities is identified as the key process to maintain decoration of island edges by impurities during growth. While the presence of impurities strongly increases the island density, a change in the scaling of island density with flux, predicted by a rate equation theory for attachment-limited growth [D. Kandel, Phys. Rev. Lett. 78, 499 (1997)], is not observed. We argue that, within the present model, even completely covered island edges do not provide efficient barriers to attachment.
\end{abstract}

Keywords: Monte Carlo simulations, Clusters, Epitaxy, Growth, Nucleation, Surface diffusion

\footnotetext{
${ }^{1}$ Corresponding author: M. Kotrla, Institute of Physics ASCR, Na Slovance 2, 18221 Praha 8, Czech Republic, tel.: +420 26605 2904; FAX +420 2 8588605, E-mail: kotrla@fzu.cz.
} 
The effect of impurities on crystal growth has been a long-standing concern in surface science [1]. Recent interest in this subject has been fueled by the prospect of using adsorbates as "surfactants" to improve the quality of epitaxially grown films [2]. A key mechanism which dramatically increases the (detrimental or beneficial) effects of adsorbates is their tendency to decorate the step edges by preferentially attaching there [3, 4]. If this induces an additional energy barrier which an adatom has to overcome to be incorporated at the step edge, the scaling exponent $\chi$ in the relation

$$
N \sim(F / D)^{\chi}
$$

between the island density $N$, the deposition flux $F$ and the adatom diffusion coefficient $D$ may be affected. Assuming a critical (= largest unstable) island size $i^{*}$, standard rate equation theory yields in two dimensions the expression [5, 自]

$$
\chi=\frac{i^{*}}{i^{*}+2}
$$

while strong incorporation barriers imply [7]

$$
\chi=\frac{2 i^{*}}{i^{*}+3}
$$

Thus inspection of the flux dependence of the island density could reveal whether or not the island edges are efficiently passivated by the adsorbates provided the critical island size is only slightly changed by the presence of adsorbates. Markov [8] has pointed out that the change from (2) to (3) can be understood as a transition from a diffusion limited to a kinetically limited growth regime.

In this paper we report on an extensive numerical study of a two-species growth model aimed at answering the following two questions: First, what kinetic processes are necessary to generate an island morphology in which the impurities decorate the island edges? Second, how does the presence of impurities affect the island density, and what role does the passivation of island edges play in this context?

We employ a full diffusion solid-on-solid model of epitaxial growth with two surface species, which we denote $A$ and $B$. We suppose that particles of type $A$ correspond to the growing material and particles of type $B$ represent the impurities. Simulations start on a flat substrate composed only of $A$ 
atoms. Both species are randomly deposited with generally different fluxes $F_{A}$ and $F_{B}$. Here, we restrict ourselves to codeposition with $F_{A}=F_{B}$. Similar results for predeposition of adsorbates (B atoms) as well as a study of the effect of the concentration of adsorbates will be presented elsewhere. The migration of each surface atom is modeled as a nearest-neighbor hopping process with rate $R_{D}=k_{0} \exp \left(-E_{D} / k_{B} T\right)$, where $k_{0}=10^{13} \mathrm{~Hz}$ is an adatom vibration frequency, $E_{D}$ is the hopping barrier, $T$ is the substrate temperature and $k_{B}$ is Boltzmann's constant. The hopping barrier is the sum of a term from the substrate $E_{\text {sub }}$ and a contribution from each lateral nearest neighbor $E_{\mathrm{n}}$. Contributions depend on local composition: For each term we have the four possibilities $A A, A B, B A$ and $B B$. Thus, the hopping barrier of adatom $X(A$ or $B)$ is

$$
E_{D}^{X}=\sum_{Y=A, B}\left(n_{0}^{Y} E_{\mathrm{sub}}^{X Y}+n_{1}^{X Y} E_{\mathrm{n}}^{X Y}\right)
$$

where $E_{\mathrm{sub}}^{X Y}$ is the hopping barrier for a free $X$ adatom on a substrate atom $Y$, $n_{0}^{Y}$ is equal to one if the substrate atom is of type $Y$ and zero otherwise, $n_{1}^{X Y}$ is the number of nearest-neighbor $X-Y$ pairs, and $E_{\mathrm{n}}^{X Y}$ is the corresponding contribution to the barrier (symmetric in $X$ and $Y$ ). Lateral interactions between impurity atoms are neglected $\left(E_{\mathrm{n}}^{B B}=0\right)$.

In the simulations reported here we used $E_{\text {sub }}^{A A}=0.8 \mathrm{eV}, E_{\mathrm{sub}}^{A B}=0.1$ $\mathrm{eV}, E_{\mathrm{sub}}^{B A}=1.0 \mathrm{eV}, E_{\mathrm{sub}}^{B B}=0.1 \mathrm{eV}$, and the substrate temperature $T=$ $500 \mathrm{~K}$. Other parameters for lateral interactions were varied. The system sizes ranged from $300 \times 300$ to $500 \times 500$. The low values of $E_{\text {sub }}^{A B}$ and $E_{\text {sub }}^{B B}$ ensure that atoms deposited on top of an impurity immediately descend to the substrate. We use fluxes in the interval from $0.00025 \mathrm{ML} / \mathrm{s}$ to $0.25 \mathrm{ML} / \mathrm{s}$. In the case of homoepitaxy, different values of $E_{\mathrm{n}}^{A A}$ correspond to different sizes of critical nucleus $i^{*}$. There are two different situations $E_{\mathrm{n}}^{A A}>E_{\mathrm{n}}^{A B}$ and $E_{\mathrm{n}}^{A A}<E_{\mathrm{n}}^{A B}$. In equilibrium at a low temperature, the former case leads to the formation of islands composed inside mainly of $A$ atoms with $B$ atoms bounded near the edges, whereas in the latter case it is energetically more favorable when $B$ atoms are inside the island. We observed, however, that for our parameters, growth always leads to intermixing of $A$ and $B$ atoms, in both cases $E_{\mathrm{n}}^{A A}>E_{\mathrm{n}}^{A B}$ and $E_{\mathrm{n}}^{A A}<E_{\mathrm{n}}^{A B}$.

Thus the energetic bias favoring segregation is not sufficient to obtain configurations with impurities mostly at island edges. To achieve this, we have to introduce an additional thermally activated process of exchange of 
an $A$ atom approaching an island edge covered with an impurity. This is a similar process to that introduced in Ref. 9] for simulation of homoepitaxy on $\mathrm{Si}(001)$ with predeposited hydrogen. Here, we allow the exchange of an $A$ atom with an impurity when it has before the exchange process at most one bond to another $A$ atom in a nearest-neighbor position. The rate of the exchange process is set to $k_{\mathrm{ex}}=k_{0} \exp \left(-E_{\mathrm{ex}} / k_{B} T\right)$, where the activation barrier $E_{\text {ex }}$ is taken to be independent of whether or not the A-atom has a nearest neighbor bond. The exchange barrier $E_{\text {ex }}$ was varied from $0.8 \mathrm{eV}$ (the diffusion barrier of free adatoms) to $2 \mathrm{eV}$. For low $E_{\mathrm{ex}}$ the impurities float on the island edges, whereas for large $E_{\text {ex }}$ they tend to get trapped inside an island. In order to obtain decorated island edges, $E_{\mathrm{ex}}$ has to be lower than a certain value which in the present case is about $1.2 \mathrm{eV}$; in the following we set $E_{\mathrm{ex}}=1 \mathrm{eV}$.

In Fig. 1a and 1b we show examples of typical configurations with the same partial coverage of both species $\theta_{A}=\theta_{B}=0.1 \mathrm{ML}$ (i.e. total coverage $\theta=\theta_{A}+\theta_{B}=0.2 \mathrm{ML}$ ) obtained by codeposition with fluxes $F_{A}=F_{B}=0.004$ $\mathrm{ML} / \mathrm{s}$. They illustrate the effect of varying the relation between $E_{\mathrm{n}}^{A A}$ and $E_{\mathrm{n}}^{A B}$ for $E_{\mathrm{n}}^{A A}=0.3 \mathrm{eV}$ (a typical configuration for homoepitaxy in shown in Fig. 1c). For $E_{\mathrm{n}}^{A B}=0.2 \mathrm{eV}$ some gaps can be seen in the impurity layer surrounding the islands, while for $E_{\mathrm{n}}^{A B}=0.4 \mathrm{eV}$ the decoration is complete. If we assume that the decorated island edge is in equilibrium with an ideal impurity gas of coverage $\theta_{B}$, then a simple detailed balance argument shows that the concentration of uncovered edge sites is $\left(1+\theta_{B} e^{E_{\mathrm{n}}^{A B} / k_{B} T}\right)^{-1}$. Thus for $T=500 \mathrm{~K}, \theta_{B}=0.1$ and $E_{\mathrm{n}}^{A B}=0.2 \mathrm{eV}$ about one tenth of the edge sites are uncovered, in accord with the visual inspection of Figure 1. Correspondingly, simulations with $E_{\mathrm{n}}^{A B}=0.1 \mathrm{eV}$ show that large portions of the step edges remain uncovered.

It can be seen from Figure 1 that the island density $N$ increases with increasing $E_{\mathrm{n}}^{A B}$. A quantitative evaluation of the dependence of island density on flux, coverage and $E_{\mathrm{n}}^{A B}$ is given in Figure 2. For comparison we show also results for homoepitaxial growth without impurities. We would like to point out three noteworthy features of these data. First, increasing the interaction energy between adatoms and impurities dramatically increases the island density. Second, the increase is essentially independent of flux, i.e. the scaling exponent $\chi$ in Eq.(1) is hardly affected by the impurities; we find $\chi=0.54$ for $E_{\mathrm{n}}^{A B}=0.2, \chi=0.45$ for $E_{\mathrm{n}}^{A B}=0.4$, and $\chi=0.54$ for homoepitaxial growth. Third, for large fluxes and large values of $E_{\mathrm{n}}^{A B}$, the island 
density continues to increase with coverage at least up to $\theta=0.2 \mathrm{ML}$.

While a detailed discussion of these effects will be left to an extended publication, here we provide a simple argument to explain why the scaling exponent (3) predicted for passivated islands is not observed in our simulations. The mechanism described by Kandel [7] relies on the rate of attachment of an adatom to an island, $S$, to be much smaller than the diffusion rate $D$ on the terrace. It is useful to introduce the probability $p=S /(D+S) \approx S / D$ for an adatom to attach during an encounter with an island edge. In the kinetically limited growth regime, characterized by (3), an adatom typically has to visit many islands before being captured. To see when this is the case, consider an adatom diffusing through an array of islands of density $N$ and linear size $R \sim \sqrt{\theta_{A} / N}$. During $n$ diffusion steps the adatom explores a region of area $\sim n$, which contains $\sim N n$ islands and $\sim N n R$ edge sites. The adatom is captured when $N n R p \approx 1$. It follows that the condition for encountering many islands prior to capture reads

$$
p \ll R^{-1} \sim \sqrt{N / \theta_{A}} .
$$

For a given value of $p$ this places a lower bound on the island density (and, according to (1), a lower bound on the flux) beyond which a crossover to conventional, diffusion limited scaling will occur.

Let us estimate $p$ for a completely decorated island. An adatom approaching the island has two options: It can return to the terrace with rate $k_{0} e^{-\left(E_{\mathrm{sub}}^{A A}+E_{\mathrm{n}}^{A B}\right) / k_{B} T}$, or it can exchange with the impurity (and thus join the island) with rate $k_{0} e^{-E_{\mathrm{ex}} / k_{B} T}$. The capture probability $p$ is the probability that the second process occurs before the first, and is given by

$$
p=\left[1+e^{\left(E_{\mathrm{ex}}-E_{\mathrm{sub}}^{A A}-E_{\mathrm{n}}^{A B}\right) / k_{B} T}\right]^{-1} .
$$

With the standard values $E_{\mathrm{ex}}=1 \mathrm{eV}, E_{\mathrm{sub}}^{A A}=0.8 \mathrm{eV}$ and $E_{\mathrm{n}}^{A B} \geq 0.2 \mathrm{eV}$ it can be seen that the condition (5) is never satisfied. To significantly decrease $p$, one would have to either increase the exchange barrier $E_{\text {ex }}$ relative to the diffusion barrier $E_{\mathrm{sub}}^{A A}$, which leads to intermixing of impurities and adatoms within the island, or to decrease the AB-interaction strength $E_{\mathrm{n}}^{A B}$, which would increase the number of gaps in the impurity layer and invalidate our assumption of complete decoration. In fact the appearance of $E_{\mathrm{n}}^{A B}$ in the exponent of (6) implies that even completely decorated island edges are not efficiently passivated in our model, because the B-atoms covering the edge 
still have "bonds" available with which to attract A-atoms and keep them near the edge long enough for an exchange to occur.

In conclusion, we have shown that the attachment-limited growth regime characterized by the scaling exponent (3) is not easily realizable in a twospecies growth system with isotropic nearest neighbor interactions. It remains to be understood what microscopic mechanism, other than passivation of island edges, is responsible for the strong increase in island density shown in Figure 2. The most obvious possibility is that the impurities reduce the mobility of adatoms by temporarily trapping them, thus effectively reducing the diffusion coefficient $D$ in (目). A detailed analysis of this mechanism will be presented elsewhere.

Acknowledgements. This work was supported by Volkswagen-Stiftung and by the COST project P3.130. 


\section{References}

[1] N. Cabrera and D.A. Vermilyea, in "Growth and Perfection of Crystals", ed. by R. Doremus, B. Roberts and D. Turnbull (Wiley, New York 1958), p. 393.

[2] D. Kandel and E. Kaxiras, The surfactant effect in semiconductor thin film growth (preprint, cond-mat/9901177).

[3] H.A. van der Vegt, H.M. van Pinxteren, M. Lohmeier, E. Vlieg and J.M.C. Thornton, Phys. Rev. Lett. 68, 3335 (1992).

[4] M. Kalff, G. Comsa and Th. Michely, Phys. Rev. Lett. 81, 1255 (1998).

[5] S. Stoyanov and D. Kashiev, in "Current Topics in Materials Science", ed. by E. Kaldis (North Holland, Amsterdam 1981), p. 69.

[6] J.A. Venables, G.D.T. Spiller and M. Hanbücken, Rep. Prog. Phys. 47, 399 (1984).

[7] D. Kandel, Phys. Rev. Lett. 78, 499 (1997).

[8] I. Markov, Phys. Rev. B 56, 12544 (1997).

[9] P. Šmilauer, K. Mizushima and D. D. Vvedensky, Phys. Rev. Lett. 81, 5600 (1998). 


\section{Figure Captions}

Fig. 1: Examples of configurations for flux $F_{A}=0.004 \mathrm{ML} / \mathrm{s}$, adatom interaction energy $E_{\mathrm{n}}^{A A}=0.3 \mathrm{eV}$, and coverage $\theta_{A}=0.1 \mathrm{ML}$ : (a) codeposition with $F_{B}=F_{A}$ and $E_{\mathrm{n}}^{A B}=0.2 \mathrm{eV}$, (b) codeposition with $F_{B}=F_{A}$ and $E_{\mathrm{n}}^{A B}=0.4 \mathrm{eV},(\mathrm{c})$ homoepitaxy $\left(F_{B}=0\right)$. We show only $50 \times 50$ sections of a larger simulation box.

Fig. 2: Averaged island density as function of flux $F_{A}$ for several values of the total coverage $\theta=\theta_{A}+\theta_{B}$ and different energy barriers: $E_{\mathrm{n}}^{A B}=0.2$ $\mathrm{eV}$ - open symbols, $E_{\mathrm{n}}^{A B}=0.4 \mathrm{eV}$ - filled symbols. The adatom interaction energy $E_{\mathrm{n}}^{A A}=0.3 \mathrm{eV}$ and the exchange barrier $E_{\mathrm{ex}}=1 \mathrm{eV}$ are fixed, and the impurity flux $F_{B}=F_{A}$. The behavior in the absence of impurities (homoepitaxy, $F_{B}=0$ ) is shown for comparison. 


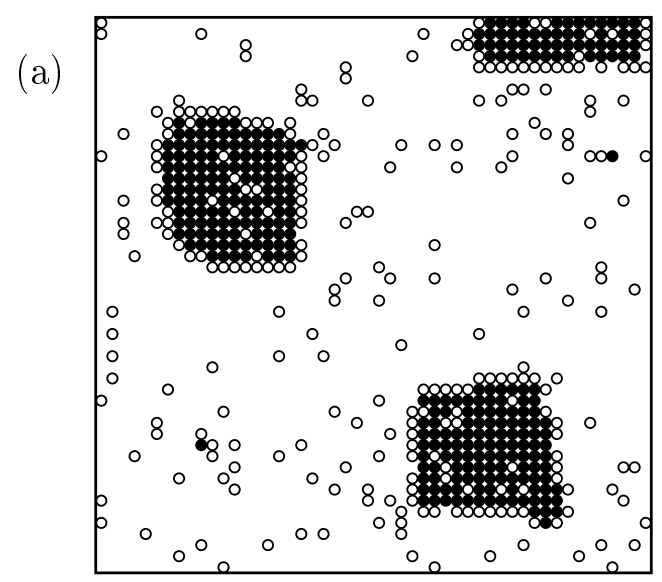

(b)

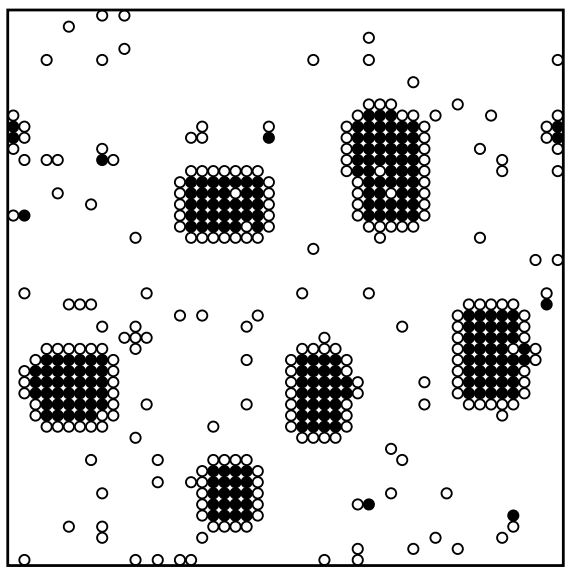

(c)

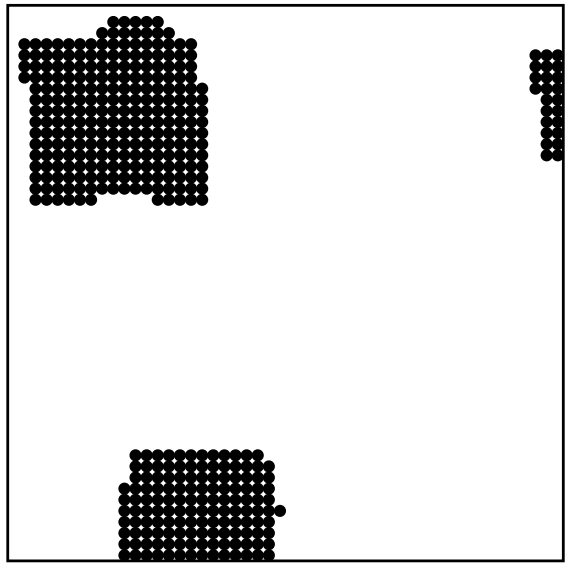

Figure 1: 


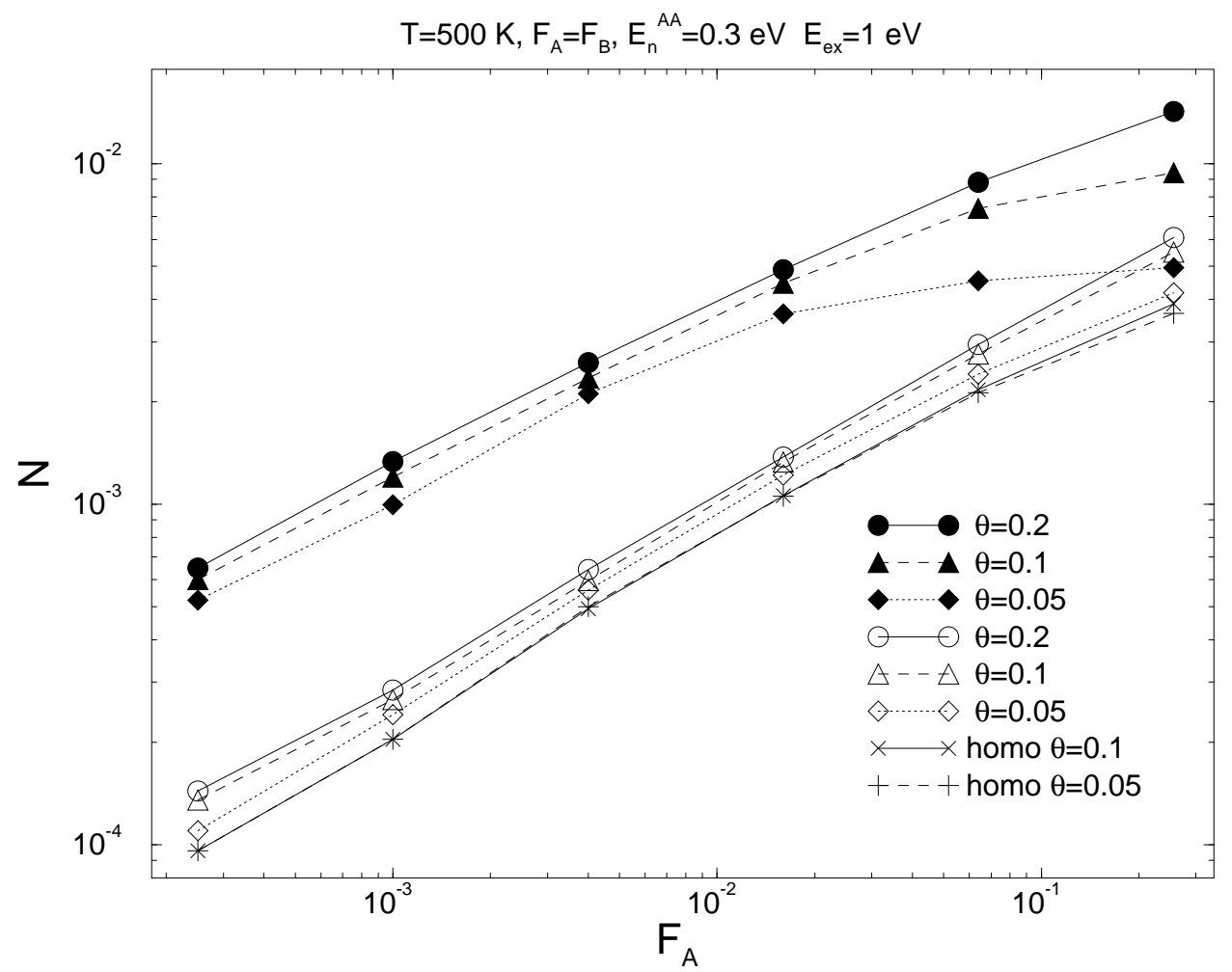

Figure 2: 